$\begin{cases}\text { JOURNAL OF ETHNOBIOLOGY } \\ \text { AND ETHNOMEDICINE }\end{cases}$

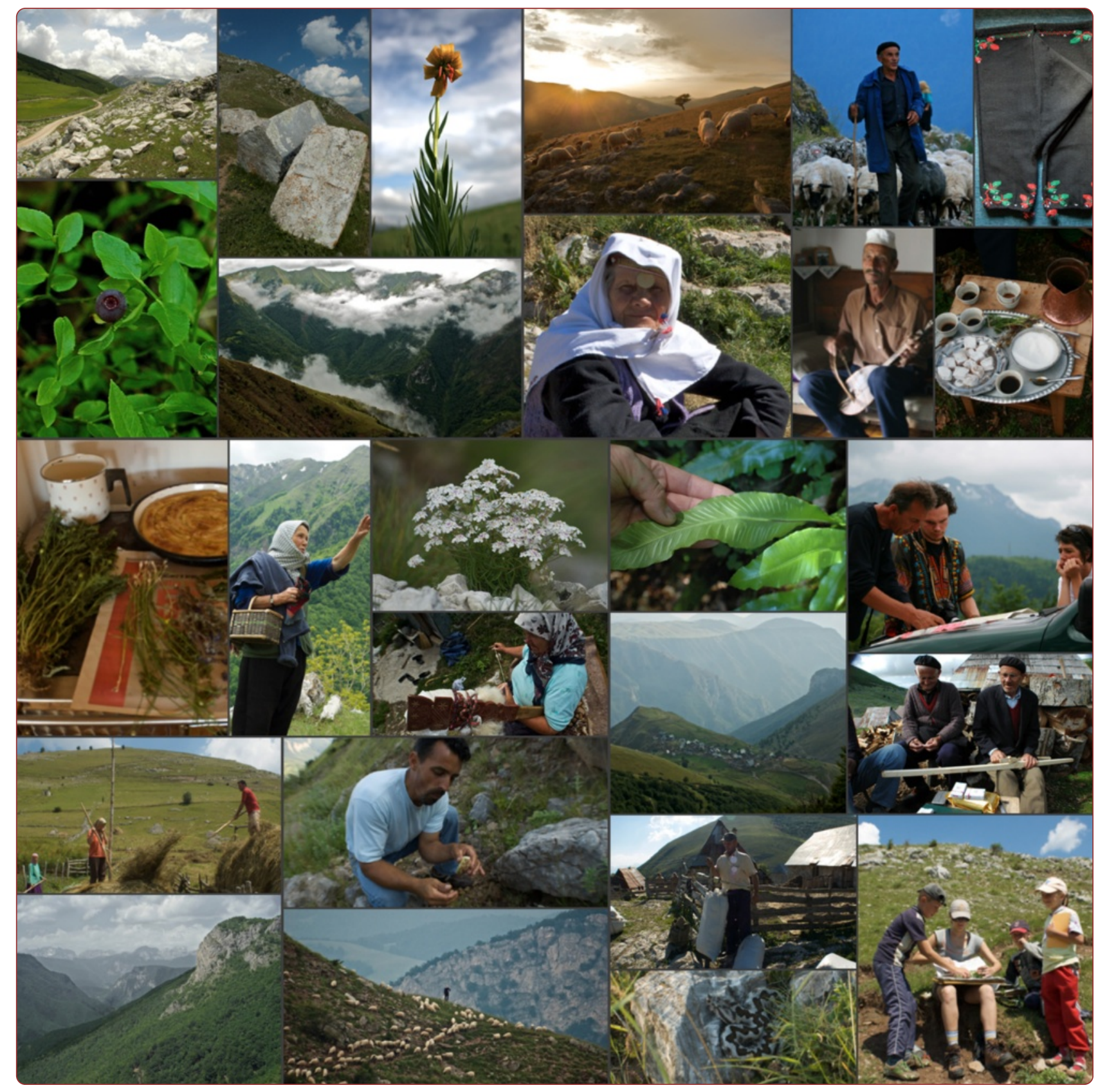

\title{
An ethnobotany of the Lukomir Highlanders of Bosnia \& Herzegovina
}

Ferrier et al. 


\title{
An ethnobotany of the Lukomir Highlanders of Bosnia \& Herzegovina
}

Jonathan Ferrier ${ }^{1,2,3,4^{*}+}$ (D), Lana Saciragic ${ }^{4,5}$, Sabina Trakic ${ }^{6 \dagger}$, Eric C. H. Chen ${ }^{1 \dagger}$, Rachelle L. Gendron ${ }^{4}$, Alain Cuerrier ${ }^{7}$, Michael J. Balick², Sulejman Redžić ${ }^{\wedge}$, Emira Alikadić $^{8}$ and John T. Arnason ${ }^{1}$

\begin{abstract}
Background: This aim of this study is to report upon traditional knowledge and use of wild medicinal plants by the Highlanders of Lukomir, Bjelašnica, Bosnia and Herzegovina (B\&H). The Highlanders are an indigenous community of approximately 60 transhumant pastoralist families who speak Bosnian (Bosanski) and inhabit a highly biodiverse region of Europe. This paper adds to the growing record of traditional use of wild plants within isolated communities in the Balkans.
\end{abstract}

Methods: An ethnobotanical study using consensus methodology was conducted in Lukomir in Bjelašnica's mountains and canyons. Field work involved individual semi-structured interviews during which informants described plants, natural product remedies, and preparation methods on field trips, garden tours, while shepherding, or in settings of their choice. Plant use categories were ranked with informant consensus factor and incorporated into a phylogenetic tree. Plants cited were compared to other ethnobotanical surveys of the country.

Results: Twenty five people were interviewed, resulting in identification of 58 species (including two subspecies) from 35 families, which were cited in 307 medicinal, 40 food, and seven material use reports. Individual plant uses had an average consensus of five and a maximum consensus of 15 out of 25. There were a number of rare and endangered species used as poisons or medicine that are endemic to Flora Europaea and found in Lukomir. Ten species (including subspecies) cited in our research have not previously been reported in the systematic ethnobotanical surveys of medicinal plant use in B\&H: (Elymus repens (L.) Gould, Euphorbia myrsinites L., Jovibarba hirta (L.) Opiz, Lilium bosniacum (Beck) Fritsch, Matricaria matricarioides (Less.) Porter ex Britton, Phyllitis scolopendrium (L.) Newman, Rubus saxatilis L., Silene uniflora Roth ssp. glareosa (Jord.) Chater \& Walters, Silene uniflora Roth ssp. prostrata (Gaudin) Chater \& Walters, Smyrnium perfoliatum L.). New uses not reported in any of the aforementioned systematic surveys were cited for a total of 28 species. Thirteen percent of medicinal plants cited are endemic: Helleborus odorus Waldst. et Kit., Gentiana lutea L., Lilium bosniacum (Beck) Fritsch, Silene uniflora Roth ssp. glareosa (Jord.) Chater \& Walters., Silene uniflora Roth ssp. prostrata (Gaudin) Chater \& Walters, Salvia officinalis L., Jovibarba hirta (L.) Opiz, and Satureja montana L. (Continued on next page)

\footnotetext{
* Correspondence: jonathanferrier@me.com

${ }^{\dagger}$ Equal contributors

Deceased

'Department of Biology, University of Ottawa, Gendron Hall Room 160, 30

Marie Curie, Ottawa K1N 6 N5ON, Canada

${ }^{2}$ Institute of Economic Botany, The New York Botanical Garden, 200th Street and Kazimiroff Blvd, The Bronx, NY 10458-5126, USA

Full list of author information is available at the end of the article
} 
(Continued from previous page)

Conclusions: These results report on the cohesive tradition of medicinal plant use among healers in Lukomir, Bosnia and Herzegovina. This work facilitates the community's development by facilitating local and international conversations about their traditional medicine and sharing insight for conservation in one of Europe's most diverse endemic floristic regions, stewarded by one of Europe's last traditional Highland peoples.

Keywords: Postwar ethnobotany, Lukomir highlanders, Bosnia and Herzegovina, Medicinal, food, and endemic plants

\section{Background}

The aim of this study is to report upon traditional knowledge and use of wild medicinal plants by the Highlanders of Lukomir, Bjelala, B, Bosnia and Herzegovina (B\&H). The project began as a collaboration with the late ethnobotanical authority in B\&H, Professor Sulejman Redžić (1954-2013) and was funded by a post war development grant from the Canadian International Development Agency (CIDA). The first ethnobotanical study published from Lukomir was contributed in Redžićs honour [1].
The project was designed to help describe Lukomir's biocultural region with the goal of assisting $B \& H$ to meet environment and health mandates of the European Union Stabilization and Association Agreement.

Lukomir is an isolated village located about $50 \mathrm{~km}$ southwest of the capital city, Sarajevo (Fig. 1). Lukomir is situated above Rakitnica canyon with only one access road, which is impassable for part of the year. This isolation has resulted in the preservation of many aspects of traditional lifestyle in Lukomir,

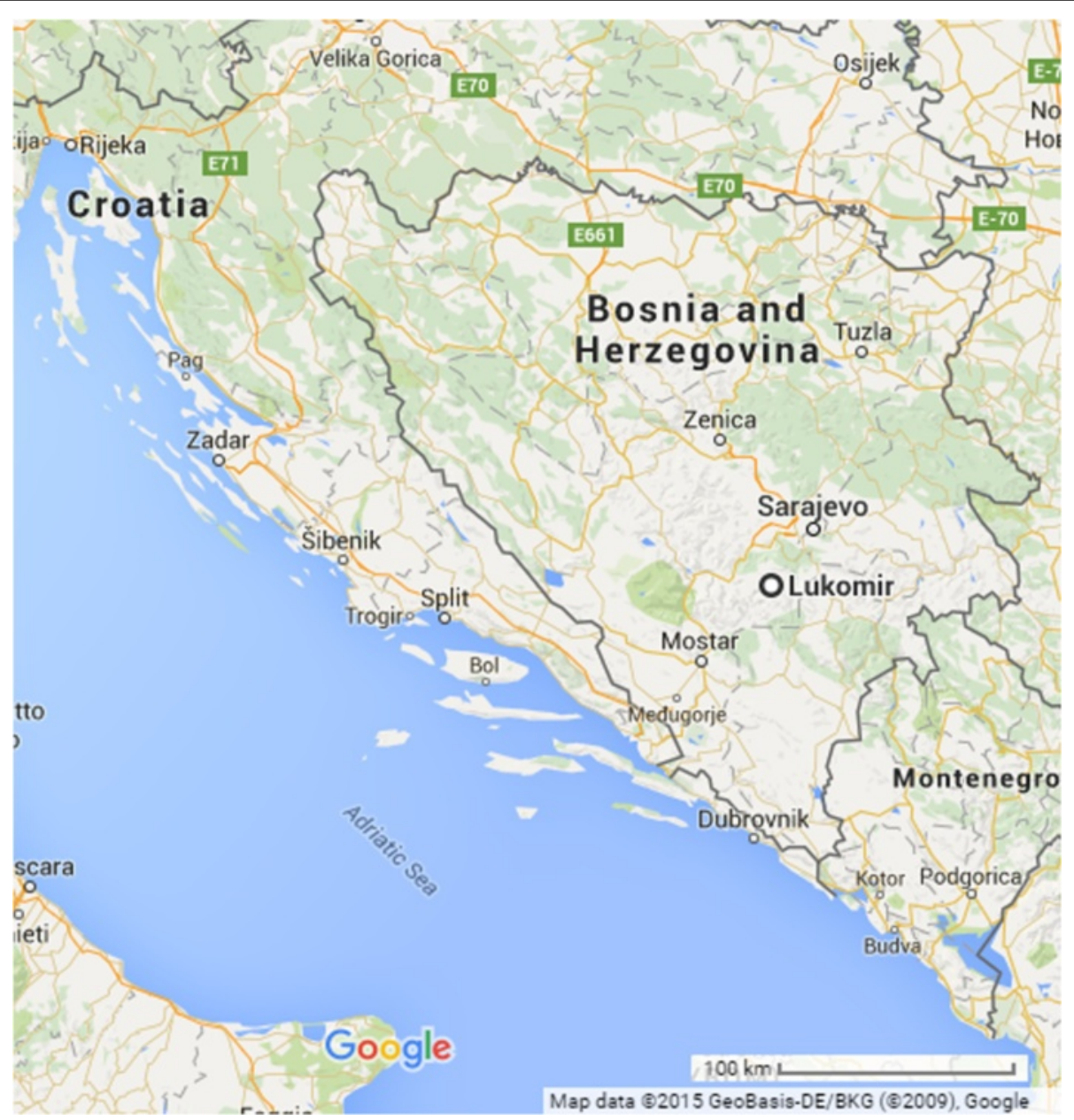

Fig. 1 Map of Bosnia and Herzegovina with Lukomir southwest of Sarajevo. Mapdata ๑ 2015 GeoBasis-DE/BKG (๑ 2009), Google 
including traditional architecture, clothing, herding and medicinal plant gathering and usage [2].

The Lukomir Highlanders are Bosniak and speak Bosnian (Bosanski). They are one of Europe's indigenous communities whose members still occasionally practice a traditional transhumant pastoralist lifestyle. They have inhabited the Lukomir territory (Figs. 1 and 2) within the Dinaric Alps for centuries and are historically Bogomil (Sulejman Redžić, personal communication). Autosomal STR loci studies link Lukomir to the isolated Adriatic islands of Brač, Hvar, and Korčula in Croatia [3]. The Highlanders also have a historic connection to transhumant peoples from the Podveležje Plateau near Mostar, B\&H, who travelled to Bjelašnica with their livestock in search of water during the summer months [4].

Until recently, shepherds with hundreds in their flocks set out on grazing journeys throughout the Lukomir territory and to neighbouring regions, especially Konjic, B\&H. In the wild, shepherds slept with their sheep flocks while other Lukomir family members gathered and cultivated for enough food and fodder to last the long winter.

Since the Bosnian War (1992-5), many inhabitants have moved to cities and herding has declined. Instead, there is a seasonal migration of former inhabitants to Lukomir to gather medicinal plants, make hay during the summer months, and carry on other traditional

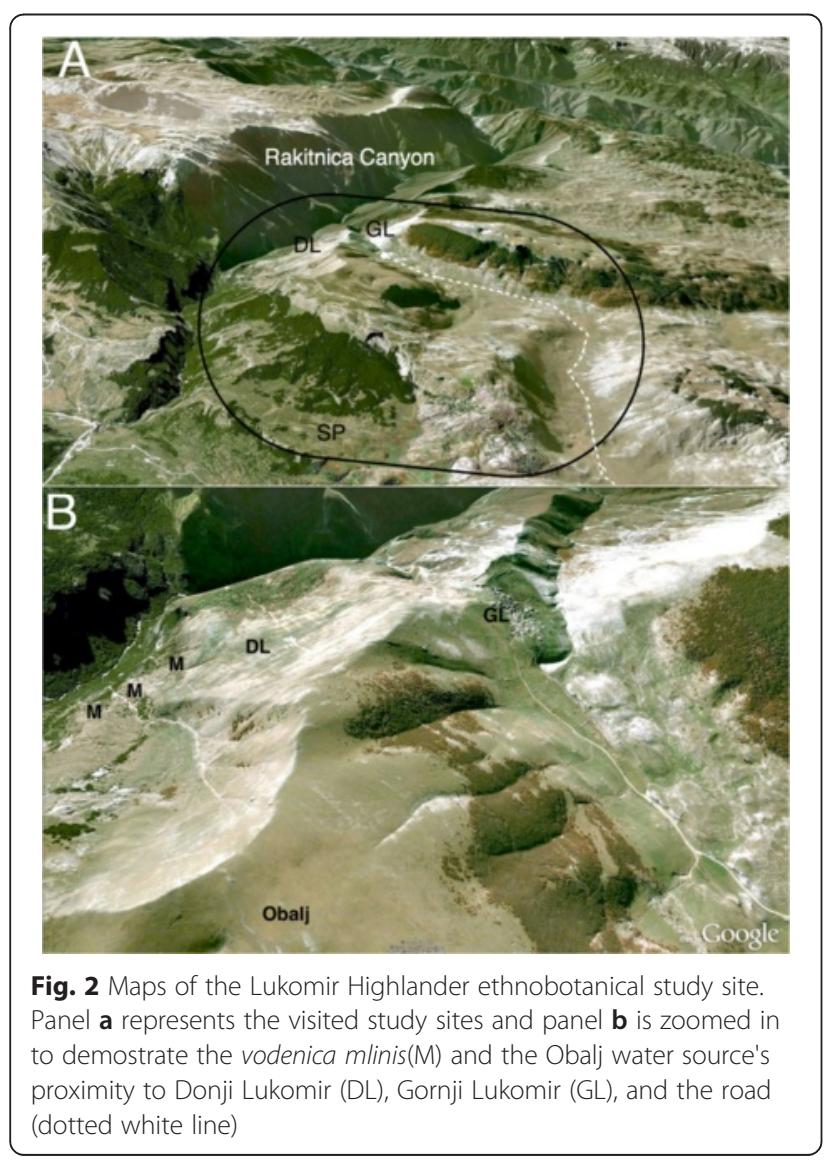

practices [2]. According to local informants, the number of people staying in Lukomir all winter and the number of people returning in the summer is declining. In the winter of 2007, three families stayed for the winter. In 2008 and 2009, only two families remained all winter. The winter of 2011-2012 was the first winter without inhabitants. This transition in Lukomir's cultural history was likely exacerbated by $15 \mathrm{~m}$ high snowdrifts, an impassable road, and relocations for work and education.

\section{Healthcare in Lukomir}

From 1950 onward, the development of the only road prompted the Highlanders to gradually relocate from Donji Lukomir (Lower Lukomir) to their current location of Gornji Lukomir (Upper Lukomir), which is now generally referred to as Lukomir (see DL and GL in Fig. 2). Before this road, Donji Lukomir provided the most efficient access to health and trade services in neighbouring regions via the Rakitnica canyon trails. Lukomir is without a primary healthcare facility, but is known to practice traditional medicine, according to Professor Sulejman Redžić.

\section{Biodiversity in Lukomir}

Approximately $60 \%$ of all vascular plant species listed in the Flora Europaea are found in the Balkan Peninsula, making Lukomir part of a highly biodiverse region of Europe $[5,6]$. Many other studies of traditional plant use for medicine and nutrition have been conducted in the Balkans, including in B\&H. Three key systemic ethnobotanical surveys of $\mathrm{B} \& H$ have recently identified hundreds of plants and thousands of preparations used in traditional human therapy [6-8]. Many of these plants are used in official pharmacopeias while others are less known and invite further ethnopharmacological studies. Many of the same uses and preparations were found across regions and ethnic groups, showing an established base of medicinal plant use. This study joins a growing collection of research whose goal is to provide an inventory and increase understanding of medicinal plants used in the Balkan Peninsula [9], including countries such as Albania [10-12], Montenegro [13], Kosovo [14], Serbia [15, 16], Bulgaria [17, 18], and Macedonia [19, 20].

\section{Methods}

Partnership, permits, and prior informed consent

This ethnobotanical study was conducted between 2007 and 2013 with the Lukomir community in Bosnia and Herzegovina, and J.F. and L.S.'s contracting agency. An international collaboration was developed with the Partnerships for Tomorrow Program, Phase II (PTP) funded by the Canadian International Development Agency (CIDA). Ferrier and Šačiragić were hosted by the University of Sarajevo and Foundation GEA+. Research permits 
were issued by the Municipality of Konjic and the University of Ottawa Human Ethics Review Board (H0509-07), with prior informed consent from Lukomir's leaders and informants. The project was compliant with Canada's tri-council guideline on research with vulnerable populations.

\section{Study site: Lukomir, Municipality of Konjic, Bosnia \& Herzegovina, Europe}

An ethnobotanical study using consensus methodology was conducted with the Lukomir Highlanders of B\&H in the highlands of Bjelašnica, located southwest of the capital city Sarajevo (Fig. 1). The Lukomir biocultural area is an alpine biogeographic region that is bordered closely by Mediterranean and Continental biogeographic regions within the biocultural area [21]. Many community members are descendants of a Bogomil lineage who first settled in Donji Lukomir (Lower Lukomir) (43.632 lat., 18.194 long., $1200 \mathrm{~m}$ a.s.l.) and eventually moved to Gornji Lukomir (Upper Lukomir, commonly referred to as Lukomir) (43.637 lat., 18.182 long., $1460 \mathrm{~m}$ a.s.l.) (Fig. 2).

We visited Lukomir repeatedly, in the summers of 2008 (June, July, August), 2009 (August), 2010 (June), and 2012 (August). By volunteering our help to the Highlanders while shepherding, collecting food, and stacking hay, we had more time for interviews and recruited 25 informants from Lukomir who described plants on mountain and canyon field trips, garden tours, while shepherding, or in settings of their choice. The informants were community healers who ranged in age from younger adults to elders. Fifty-six percent were women. Notes and photos were taken when participants chose to display preparation methods of plant and natural remedies. Plant vouchers, the authors' working field guide, and an iPad (Apple, Cupertino, USA) were used to display collections and photos to elders who could not venture over the mountainside, or for informant review purposes [22].

Land mines were avoided by consulting with a map from the Bosnia and Herzegovina Mine Action Center [23]. Only parts of the Lukomir territory were surveyed by BH MAC and so all plants were collected on trail sides or in areas that were constantly travelled by sheep herds.

Field work followed consensus methodology with individual semi-structured interviews during which L.S., J.F., and S.R. collected the following data: (1) specimen voucher number, (2) photo number, (3) common name, (4) scientific name, (5) family, (6) GPS coordinates, (7) altitude (m a.s.l.), (8) habitat, (9) sub-habitat, (10) flowering time and description, (11) medically active collection time, (12) use, (13) use category, (14) plant part used, (15) amount used, (16) medicine preparation method,
(17) medicine administration method, (18) medicine dosing regimen, (19) ethnographic details, (20) informant name and number, and (21) date. Duplicate vouchers were collected (when sustainable) for deposition at the University of Sarajevo Herbarium and the University of Ottawa herbarium, OTT (Ferrier 351-458).

While this work was focused on medical plant use, a non-exhaustive record of food and material uses was made in association with the medicinal plants. Due to the expense and time associated with ethnobotanical projects, we felt that including the food and material mentions would be of greater value to the Lukomir community.

\section{Ethnobotanical analysis and statistics Human selection of plants}

Consensus on an individual usage was the number of informants using the plant out of 25. For categories of phytochemical and pharmacological interest, the informant consensus factor $\left(F_{i c}\right)$ function, created by Trotter and Logan [24], was used to supply a numerical ranking factor based on the number of use reports $\left(n_{u r}\right)$ and the number of taxa $\left(n_{\text {taxa }}\right)$ per usage category.

$$
F_{i c}=\left(n_{u r}-n_{\text {taxa }}\right) /\left(n_{u r}-1\right)
$$

\section{Statistics and phylogenetic tree}

Statistical analysis was conducted using Prism 6 software. A phylogenetic tree was constructed using TreeGraph 2.0.47-206 beta, FigTree v1.4.0 (Fig. 3). Taxa are based on the flora of $\mathrm{B} \& \mathrm{H}$ [25] and corrected to match accepted names in Tropicos [26] and the topology presented is based on Angiosperm Phylogeny Group III [27].

\section{Results and discussion Interviews}

During our interviews, the Highlanders identified 58 species (including 2 subspecies) from 35 families, which were cited in 307 medicinal, 40 food, and seven material use reports and are included Table 1. Grassland habitats generated the most use reports of plants. Medicinal plant collection was greatest from mid-July to early August as many healers collected plants while in the grasslands harvesting hay.

The average consensus on use of medicinal plants was 3.5 informants out of 25 , and a maximum consensus of 15 was obtained on the medicinal plants Mentha longifolia (L.) L. and Salvia officinalis L. Average food plant consensus was three. The maximum food plant consensus was eight for Urtica dioica L. Finally, there was a maximum consensus of two for material plants Pinus nigra Arnold and Cornus mas L. 


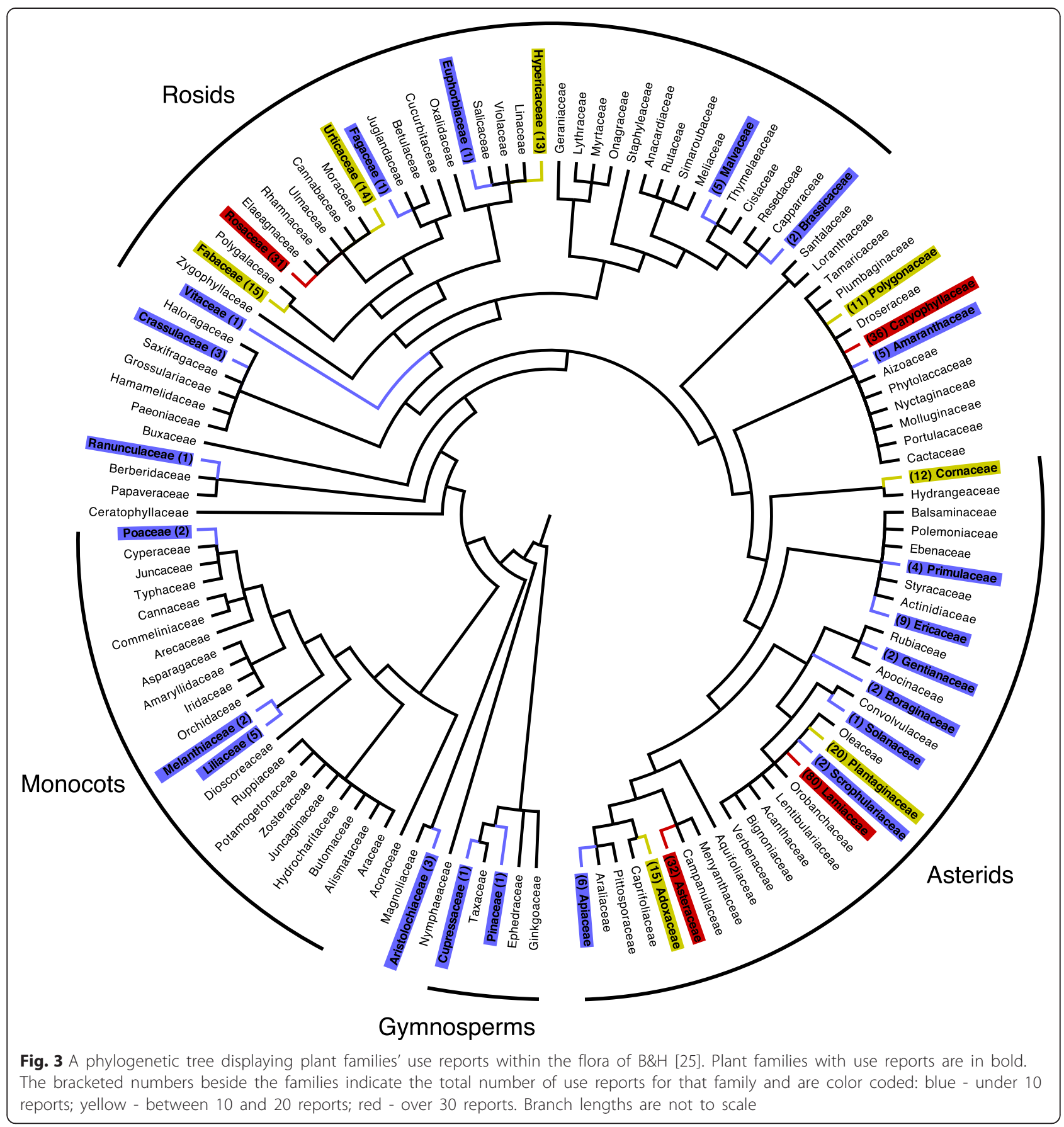

For a community of approximately 60 households, almost half of the households were interviewed. We wondered if the list of medicinal plants was small; however, when looking at our cross-cultural comparison of Cree, Highlanders and Maya we noted that while Lukomir had fewer use reports than the Maya, they had much more than the Cree [28]. This made sense to us that the Cree had less than Lukomir because of their northern location, less biodiversity and cultural assimilation via residential schools in Canada, and that Lukomir healers had fewer reports than the Maya because the Maya live in a tropical rain forest with much greater biodiversity. Lukomir's data, within a continental, alpine and Mediterranean geography in the middle of these two extremes felt reasonable to us.

\section{Frequency of use reports per usage category}

The number of use reports per usage category (Fig. 4) varied from 1 to 72 . High frequency categories included: genitourinary system disorders, panacea, pain, 
Table 1 Medicinal, food, and material taxa used by the Lukomir Highlanders of Bosnia \& Herzegovina

\begin{tabular}{|c|c|c|c|c|c|c|c|}
\hline Family & Genus species & $\dagger *$ & Common name & Habitat & Syntaxa & $f$ & $\mathrm{VN}$ \\
\hline Adoxaceae & Sambucus nigra $\mathrm{L}$. & & Zova, Zobovina, Elder & $\begin{array}{l}\text { Village \& } \\
\text { shepherd trails }\end{array}$ & $\mathrm{F}$ & 11 & 354 \\
\hline Adoxaceae & Sambucus wightiana Wall. ex Wight \& Arn. & & Haptovina & $\begin{array}{l}\text { Mountainside } \\
\text { slope }\end{array}$ & Ea, Art & 1 & 376 \\
\hline Adoxaceae & Sambucus wightiana Wall. ex Wight \& Arn. & & Haptovina & $\begin{array}{l}\text { Mountainside } \\
\text { slope }\end{array}$ & Ea, Art & 3 & 376 \\
\hline Amaranthaceae & Chenopodium bonus-henricus $\mathrm{L}$. & & Šćir, Good King Henry & $\begin{array}{l}\text { Village \& } \\
\text { shepherd trails }\end{array}$ & $\mathrm{Ch}, \mathrm{O}$ & 5 & 370 \\
\hline Apiaceae & Smyrnium perfoliatum L. & & Ljaljica, Perfoliate alexanders & Grassland & O, Arr & 2 & 380 \\
\hline Apiaceae & Smyrnium perfoliatum L. & & Ljaljica, Perfoliate alexanders & Grassland & O, Arr & 2 & 380 \\
\hline Apiaceae & Smyrnium perfoliatum L. & & Ljaljica, Perfoliate alexanders & Grassland & O, Arr & 2 & 38 \\
\hline Aristolochiaceae & Asarum europaeum $\mathrm{L}$. & & $\begin{array}{l}\text { Kopitnik/Kopitnjak, European wild } \\
\text { ginger }\end{array}$ & $\begin{array}{l}\text { Deciduous } \\
\text { forest }\end{array}$ & F, Qp, O-Co & 1 & $3 \varepsilon$ \\
\hline Aristolochiaceae & Asarum europaeum $\mathrm{L}$. & & $\begin{array}{l}\text { Kopitnik/Kopitnjak, European wild } \\
\text { ginger }\end{array}$ & $\begin{array}{l}\text { Deciduous } \\
\text { forest }\end{array}$ & F, Qp, O-Co & 2 & 382 \\
\hline Aspleniaceae & Phyllitis scolopendrium (L.) Newman & & $\begin{array}{l}\text { Podrebnica (male and female) } \\
\text { indicated by sori }\end{array}$ & Riparian & F, Amph & 10 & 357 \\
\hline Asteraceae & Achillea millefolium $\mathrm{L}$. & & Kunica, Yarrow & $\begin{array}{l}\text { Village \& } \\
\text { shepherd trails }\end{array}$ & Arr, Pm, Ch & 9 & 358 \\
\hline Asteraceae & Achillea millefolium $\mathrm{L}$. & & Kunica, Yarrow & $\begin{array}{l}\text { Village \& } \\
\text { shepherd trails }\end{array}$ & Arr, Pm, Ch & 1 & 358 \\
\hline Asteraceae & Artemisia absinthium L. & & Pelin, Absinthe wormwood & $\begin{array}{l}\text { Mountainside } \\
\text { slope }\end{array}$ & Art, O & 1 & 3 \\
\hline Asteraceae & Cichorium intybus $\mathrm{L}$. & & Konjanik, Chicory & $\begin{array}{l}\text { Village \& } \\
\text { shepherd trails }\end{array}$ & Art, $\mathrm{O}, \mathrm{Ag}$ & 1 & 4 \\
\hline Asteraceae & $\begin{array}{l}\text { Matricaria matricarioides (Less.) Porter ex } \\
\text { Britton }\end{array}$ & & Kamilica, Stomaklija, Pineappleweed & $\begin{array}{l}\text { Village \& } \\
\text { shepherd trails }\end{array}$ & $\mathrm{Pm}$ & 1 & 351 \\
\hline Asteraceae & $\begin{array}{l}\text { Matricaria matricarioides (Less.) Porter ex } \\
\text { Britton }\end{array}$ & & Kamilica, Stomaklija, Pineappleweed & $\begin{array}{l}\text { Village \& } \\
\text { shepherd trails }\end{array}$ & $\mathrm{Pm}$ & 1 & 397 \\
\hline Asteraceae & $\begin{array}{l}\text { Matricaria matricarioides (Less.) Porter ex } \\
\text { Britton }\end{array}$ & & Kamilica, Stomaklija, Pineappleweed & $\begin{array}{l}\text { Village \& } \\
\text { shepherd trails }\end{array}$ & $\mathrm{Pm}$ & 12 & 351 \\
\hline Asteraceae & $\begin{array}{l}\text { Matricaria matricarioides (Less.) Porter ex } \\
\text { Britton }\end{array}$ & & Kamilica, Stomaklija, Pineappleweed & $\begin{array}{l}\text { Village \& } \\
\text { shepherd trails }\end{array}$ & $\mathrm{Pm}$ & 1 & 35 \\
\hline Asteraceae & Taraxacum officinale F.H. Wigg. & & Maslačak, Dandelion & $\begin{array}{l}\text { Village \& } \\
\text { shepherd trails }\end{array}$ & $\begin{array}{l}\text { Arr, O, Ag, } \\
\mathrm{Pm}\end{array}$ & 1 & 412 \\
\hline Asteraceae & Tussilago farfara $\mathrm{L}$. & & Podbijel ( $\widehat{o}$ or P), Coltsfoot & Riparian & $\mathrm{O}, \mathrm{Pm}$ & 4 & 371 \\
\hline Boraginaceae & Symphytum officinale $\mathrm{L}$. & & Gavez, Common comfrey, Boneset & Riparian & $\mathrm{Ag}, \mathrm{Bid}, \mathrm{Art}$ & 2 & 38 \\
\hline Brassicaceae & Capsella bursa-pastoris (L.) Medik. & & Rustemača, Shepherd's purse & $\begin{array}{l}\text { Village \& } \\
\text { shepherd trails }\end{array}$ & Ch, O, Art & 2 & 398 \\
\hline Caryophyllaceae & $\begin{array}{l}\text { Silene uniflora Roth ssp. glareosa (Jord.) } \\
\text { Chater \& Walters }\end{array}$ & $\dagger$ & Puca, Sea campion & Grassland & Arr, Be, O-Co & 2 & 353 \\
\hline Caryophyllaceae & $\begin{array}{l}\text { Silene uniflora Roth ssp. glareosa (Jord.) } \\
\text { Chater \& Walters }\end{array}$ & $\dagger$ & Puca, Sea campion & Grassland & Arr, Be, O-Co & 7 & 353 \\
\hline Caryophyllaceae & $\begin{array}{l}\text { Silene uniflora Roth ssp. glareosa (Jord.) } \\
\text { Chater \& Walters }\end{array}$ & $\dagger$ & Puca, Sea campion & Grassland & Arr, Be, O-Co & 1 & 353 \\
\hline Caryophyllaceae & $\begin{array}{l}\text { Silene uniflora Roth ssp. glareosa (Jord.) } \\
\text { Chater \& Walters }\end{array}$ & $\dagger$ & Puca, Sea campion & Grassland & Arr, Be, O-Co & 8 & 353 \\
\hline Caryophyllaceae & $\begin{array}{l}\text { Silene uniflora Roth ssp. prostrata (Gaudin) } \\
\text { Chater \& Walters }\end{array}$ & $\dagger$ & Puca, Sea campion & Grassland & Arr, Be, O-Co & 8 & 350 \\
\hline Caryophyllaceae & $\begin{array}{l}\text { Silene uniflora Roth ssp. prostrata (Gaudin) } \\
\text { Chater \& Walters }\end{array}$ & $\dagger$ & Puca, Sea campion & Grassland & Arr, Be, O-Co & 2 & 350 \\
\hline Caryophyllaceae & Silene uniflora Roth ssp. prostrata (Gaudin) & $\dagger$ & Puca, Sea campion & Grassland & Arr, Be, O-Co & 7 & 350 \\
\hline
\end{tabular}


Table 1 Medicinal, food, and material taxa used by the Lukomir Highlanders of Bosnia \& Herzegovina (Continued)

\begin{tabular}{|c|c|c|c|c|c|c|c|}
\hline Caryophyllaceae & $\begin{array}{l}\text { Silene uniflora Roth ssp. prostrata (Gaudin) } \\
\text { Chater \& Walters }\end{array}$ & $\dagger$ & Puca, Sea campion & Grassland & Arr, Be, O-Co & 1 & 350 \\
\hline Cornaceae & Cornus mas L. & & Drijen, European dogwood & $\begin{array}{l}\text { Deciduous } \\
\text { forest }\end{array}$ & $\begin{array}{l}\text { O-Co, } \\
\text { F,Qp,Ps }\end{array}$ & 2 & 384 \\
\hline Cornaceae & Cornus mas L. & & Drijen, European dogwood & $\begin{array}{l}\text { Deciduous } \\
\text { forest }\end{array}$ & $\begin{array}{l}\text { O-Co, } \\
\text { F,Qp,Ps }\end{array}$ & 2 & 384 \\
\hline Cornaceae & Cornus mas L. & & Drijen, European dogwood & $\begin{array}{l}\text { Deciduous } \\
\text { forest }\end{array}$ & $\begin{array}{l}\text { O-Co, } \\
\text { F,Qp,Ps }\end{array}$ & 2 & 384 \\
\hline Cornaceae & Cornus mas L. & & Drijen, European dogwood & $\begin{array}{l}\text { Deciduous } \\
\text { forest }\end{array}$ & $\begin{array}{l}\text { O-Co, } \\
\text { F,Qp,Ps }\end{array}$ & 2 & 384 \\
\hline Cornaceae & Cornus mas L. & & Drijen, European dogwood & $\begin{array}{l}\text { Deciduous } \\
\text { forest }\end{array}$ & $\begin{array}{l}\text { O-Co, } \\
\text { F,Qp,Ps }\end{array}$ & 2 & 384 \\
\hline Cornaceae & Cornus mas L. & & Drijen, European dogwood & $\begin{array}{l}\text { Deciduous } \\
\text { forest }\end{array}$ & $\begin{array}{l}\text { O-Co, } \\
\text { F,Qp,Ps }\end{array}$ & 2 & 384 \\
\hline Crassulaceae & Jovibarba hirta (L.) Opiz & $\dagger$ & $\begin{array}{l}\text { Čuvarkuća, Beard of Jupiter, Hen and } \\
\text { Eggs }\end{array}$ & Rockland & Amph & 1 & 379 \\
\hline Crassulaceae & Jovibarba hirta (L.) Opiz & $\dagger$ & $\begin{array}{l}\text { Čuvarkuća, Beard of Jupiter, Hen and } \\
\text { Eggs }\end{array}$ & Rockland & Amph & 1 & 379 \\
\hline Crassulaceae & Sedum sexangulare $\mathrm{L}$. & & Zednjak, Tasteless stonecrop & Rockland & Amph & 1 & 416 \\
\hline Cupressaceae & Juniperus communis L. & & Smreka, Common juniper & $\begin{array}{l}\text { Mountainside } \\
\text { slope }\end{array}$ & Jun, O-Co & 1 & 399 \\
\hline Equisetaceae & Equisetum arvense $\mathrm{L}$. & & Preslica, Field horsetail & Riparian & $\mathrm{Pm}, \mathrm{Ch}$ & 3 & 367 \\
\hline Equisetaceae & Equisetum arvense $\mathrm{L}$. & & Preslica, Field horsetail & Riparian & $\mathrm{Pm}, \mathrm{Ch}$ & 2 & 367 \\
\hline Ericaceae & Vaccinium myrtillus L. & & Borovnica, European blueberry & $\begin{array}{l}\text { Mountainside } \\
\text { slope }\end{array}$ & $V, V-P$ & 1 & 368 \\
\hline Ericaceae & Vaccinium myrtillus L. & & Borovnica, European blueberry & $\begin{array}{l}\text { Mountainside } \\
\text { slope }\end{array}$ & V, V-P & 5 & 368 \\
\hline Ericaceae & Vaccinium vitis-idaea $\mathrm{L}$. & & Brusnica, Lingonberry & $\begin{array}{l}\text { Mountainside } \\
\text { slope }\end{array}$ & V & 1 & 385 \\
\hline Ericaceae & Vaccinium vitis-idaea $\mathrm{L}$. & & Brusnica, Lingonberry & $\begin{array}{l}\text { Mountainside } \\
\text { slope }\end{array}$ & V & 2 & 385 \\
\hline Euphorbiaceae & Euphorbia myrsinites L. & & Mliječavac, Myrtle spurge & Rockland & Af, S-Ch, Be & 1 & 400 \\
\hline Fabaceae & Anthyllis vulneraria $\mathrm{L}$. & & Ranjenik, Kidney vetch & Grassland & $\mathrm{Be}, \mathrm{St}$ & 4 & 372 \\
\hline Fabaceae & Anthyllis vulneraria $\mathrm{L}$. & & Ranjenik, Kidney vetch & Grassland & $\mathrm{Be}, \mathrm{St}$ & 4 & 372 \\
\hline Fabaceae & Ononis spinosa $\mathrm{L}$. & & Gladišika, Spiny restharrow & Grassland & $\mathrm{Be}, \mathrm{S}-\mathrm{Ch}, \mathrm{Ps}$ & 3 & 377 \\
\hline Fabaceae & Trifolium pratense L. & & Crvena djetelina, Red clover & Grassland & Arr, Ag, Be & 2 & 386 \\
\hline Fabaceae & Trifolium repens $\mathrm{L}$. & & Bijela djetelina, White clover & Grassland & Ag, M, Arr & 2 & 387 \\
\hline Fagaceae & Fagus sylvatica $\mathrm{L}$. & & Bukva, European beech & $\begin{array}{l}\text { Deciduous } \\
\text { forest }\end{array}$ & $\mathrm{F}$ & 1 & 410 \\
\hline Gentianaceae & Gentiana lutea L. & t & Lincura, Great yellow gentian & Grassland & St, Pim & 1 & 393 \\
\hline Gentianaceae & Gentiana lutea L. & $\dagger$ & Lincura, Great yellow gentian & Grassland & St, Pim & 1 & 393 \\
\hline Hypericaceae & Hypericum perforatum L. & & Kantarion, Ženska trava, St. John's wort & Grassland & Orig, Ps, Be & 2 & 355 \\
\hline Hypericaceae & Hypericum perforatum L. & & Kantarion, Ženska trava, St. John's wort & Grassland & Orig, Ps, Be & 11 & 355 \\
\hline Lamiaceae & Mentha longifolia (L.) L. & & Metvica, Nana, Horse mint & Riparian & $\begin{array}{l}\text { Bid, Mol, } \\
\text { M-C }\end{array}$ & 1 & 413 \\
\hline Lamiaceae & Mentha longifolia (L.) L. & & Metvica, Nana, Horse mint & Riparian & $\begin{array}{l}\text { Bid, Mol, } \\
\text { M-C }\end{array}$ & 1 & 401 \\
\hline Lamiaceae & Mentha longifolia (L.) L. & & Metvica, Nana, Horse mint & Riparian & $\begin{array}{l}\text { Bid, Mol, } \\
\text { M-C }\end{array}$ & 15 & 349 \\
\hline Lamiaceae & Mentha pulegium L. & & Verem, European pennyroyal & Riparian & $\begin{array}{l}\text { Bid, Mol, } \\
\text { M-C }\end{array}$ & 1 & 402 \\
\hline
\end{tabular}


Table 1 Medicinal, food, and material taxa used by the Lukomir Highlanders of Bosnia \& Herzegovina (Continued)

\begin{tabular}{|c|c|c|c|c|c|c|c|}
\hline Lamiaceae & Nepeta cataria L. & & Macina trava, Catnip & Rockland & $\mathrm{O}, \mathrm{Be}$ & 5 & 369 \\
\hline Lamiaceae & Nepeta cataria L. & & Macina trava, Catnip & Rockland & $\mathrm{O}, \mathrm{Be}$ & 5 & 369 \\
\hline Lamiaceae & Nepeta cataria L. & & Macina trava, Catnip & Rockland & $\mathrm{O}, \mathrm{Be}$ & 5 & 369 \\
\hline Lamiaceae & Salvia officinalis $\mathrm{L}$. & $\dagger$ & Kadulja, Sage & $\begin{array}{l}\text { Mountainside } \\
\text { slope }\end{array}$ & $\mathrm{S}-\mathrm{Ch}, \mathrm{O}-\mathrm{Co}$ & 15 & 348 \\
\hline Lamiaceae & Salvia officinalis $L$. & t & Kadulja, Sage & $\begin{array}{l}\text { Mountainside } \\
\text { slope }\end{array}$ & $\mathrm{S}-\mathrm{Ch}, \mathrm{O}-\mathrm{Co}$ & 1 & 348 \\
\hline Lamiaceae & Satureja montana L. & & Vrijesak, Winter savory & Rockland & $\mathrm{S}-\mathrm{Ch}, \mathrm{O}-\mathrm{Co}$ & 6 & 366 \\
\hline Lamiaceae & Satureja montana L. & t & Vrijesak, Winter savory & Rockland & $\mathrm{S}-\mathrm{Ch}, \mathrm{O}-\mathrm{Co}$ & 1 & 366 \\
\hline Lamiaceae & Teucrium montanum L. & & Iva, Mountain germander & Rockland & $\mathrm{Be}, \mathrm{S}-\mathrm{Ch}$ & 12 & 352 \\
\hline Lamiaceae & Thymus serpyllum L. & & $\begin{array}{l}\text { Čubra (Crvena i Bijela), Majčina dušica, } \\
\text { Breckland thyme }\end{array}$ & Rockland & $\mathrm{Be}, \mathrm{S}-\mathrm{Ch}$ & 5 & 365 \\
\hline Lamiaceae & Thymus serpyllum L. & & $\begin{array}{l}\text { Čubra (Crvena i Bijela), Majčina dušica, } \\
\text { Breckland thyme }\end{array}$ & Rockland & $\mathrm{Be}, \mathrm{S}-\mathrm{Ch}$ & 7 & 365 \\
\hline Liliaceae & Convallaria majalis $\mathrm{L}$. & & Dudurika, Lily of the vally & $\begin{array}{l}\text { Deciduous } \\
\text { forest }\end{array}$ & Qp, F, O-Co & 3 & 378 \\
\hline Liliaceae & Lilium bosniacum (Beck) Fritsch & t & Bosanski ljiljan, Zlatan, Bosnian lily & Grassland & Arr, Be, O-Co & 1 & 417 \\
\hline Liliaceae & Lilium bosniacum (Beck) Fritsch & $\dagger$ & Bosanski ljiljan, Zlatan, Bosnian lily & Grassland & Arr, Be, O-Co & 1 & 417 \\
\hline Malvaceae & Tilia platyphyllos Scop. & & Lipa, Large-leaved linden & Grassland & $F, Q p$ & 4 & 374 \\
\hline Malvaceae & Tilia platyphyllos Scop. & & Lipa, Large-leaved linden & Grassland & $F, Q p$ & 1 & 374 \\
\hline Melanthiaceae & Veratrum album $\mathrm{L}$. & & Čemerika, European white hellebore & Grassland & Ad, Arr & 2 & 388 \\
\hline Parmeliaceae & Cetraria islandica (L.) Ach & & Islandski lišaj, Mašina, Iceland moss & $\begin{array}{l}\text { Mountainside } \\
\text { slope }\end{array}$ & St, Be & 1 & 403 \\
\hline Pinaceae & Pinus nigra Arnold & & Bor, Black pine & $\begin{array}{l}\text { Mountainside } \\
\text { slope }\end{array}$ & St, Be & 1 & 418 \\
\hline Plantaginaceae & Plantago lanceolata $\mathrm{L}$. & & Bokvica + , Ribwort plantain & Grassland & Arr, Ag, Be & 1 & 359 \\
\hline Plantaginaceae & Plantago lanceolata $\mathrm{L}$. & & Bokvica + , Ribwort plantain & Grassland & Arr, Ag, Be & 9 & 359 \\
\hline Plantaginaceae & Plantago major L. & & Bokvica, Broadleaf plantain & $\begin{array}{l}\text { Village \& } \\
\text { shepherd trails }\end{array}$ & $\mathrm{Pm}, \mathrm{Ch}$ & 1 & 360 \\
\hline Plantaginaceae & Plantago major L. & & Bokvica, Broadleaf plantain & $\begin{array}{l}\text { Village \& } \\
\text { shepherd trails }\end{array}$ & $\mathrm{Pm}, \mathrm{Ch}$ & 9 & 360 \\
\hline Poaceae & Elymus repens (L.) Gould & & Pirika, Couch grass & Grassland & Pm, Ag, Arr & 1 & 389 \\
\hline Poaceae & Elymus repens (L.) Gould & & Pirika, Couch grass & Grassland & Pm, Ag, Arr & 1 & 389 \\
\hline Polygonaceae & Polygonum bistorta $\mathrm{L}$. & & Srčanik, Bistort & Grassland & Ad, Arr & 11 & 356 \\
\hline Primulaceae & Primula veris $\mathrm{L}$. & & Jagorčevina, Cowslip & Grassland & Arr, Ad, Ps & 4 & 373 \\
\hline Ranunculaceae & Helleborus odorus Waldst. \& Kit. ex Willd. & $\dagger$ & Kukurijek, Hellebore & Grassland & $\begin{array}{l}\text { Ps, Cor, F, } \\
\mathrm{Qp}\end{array}$ & 1 & 394 \\
\hline Rosaceae & Crataegus monogyna Jacq. & & Glog, Gloginje, Hawthorn & $\begin{array}{l}\text { Deciduous } \\
\text { forest }\end{array}$ & Ps, Cor, Qp & 2 & 361 \\
\hline Rosaceae & Crataegus monogyna Jacq. & & Glog, Gloginje, Hawthorn & $\begin{array}{l}\text { Deciduous } \\
\text { forest }\end{array}$ & Ps, Cor, Qp & 8 & 361 \\
\hline Rosaceae & Prunus spinosa $\mathrm{L}$. & & Trn, Trnjina, Blackthorne Rakija, Brandy & $\begin{array}{l}\text { Mountainside } \\
\text { slope }\end{array}$ & Ps, F, Qp & 1 & 405 \\
\hline Rosaceae & Prunus spinosa $\mathrm{L}$. & & Trn, Trnjina, Blackthorne Rakija, Brandy & $\begin{array}{l}\text { Mountainside } \\
\text { slope }\end{array}$ & $P s, F, Q p$ & 1 & 405 \\
\hline Rosaceae & Prunus spinosa $\mathrm{L}$. & & Trn, Trnjina, Blackthorne Rakija, Brandy & $\begin{array}{l}\text { Mountainside } \\
\text { slope }\end{array}$ & $P s, F, Q p$ & 1 & 405 \\
\hline Rosaceae & Rosa glauca Pourr. & & Šipak/Šipina & $\begin{array}{l}\text { Mountainside } \\
\text { slope }\end{array}$ & O-Co, Orig & 8 & 364 \\
\hline Rosaceae & Rosa glauca Pourr. & & Šipak/Šipina, Redleaf rose & $\begin{array}{l}\text { Mountainside } \\
\text { slope }\end{array}$ & O-Co, Orig & 8 & 364 \\
\hline
\end{tabular}


Table 1 Medicinal, food, and material taxa used by the Lukomir Highlanders of Bosnia \& Herzegovina (Continued)

\begin{tabular}{|c|c|c|c|c|c|c|c|}
\hline Rosaceae & Rubus idaeus L. & & Malina, Raspberry & $\begin{array}{l}\text { Deciduous } \\
\text { forest }\end{array}$ & Ea, Atr & 1 & 406 \\
\hline Rosaceae & Rubus saxatilis L. & & Kupina, Stone bramble & Grassland & Af, S-Ch & 1 & 407 \\
\hline Scrophulariaceae & Verbascum thapsus L. & & Divizbina, Divizma, Great mullein & Grassland & Art, $\mathrm{O}$ & 2 & 390 \\
\hline Solanaceae & Solanum tuberosum L. & $*$ & Krompir, Potato & Cultivated & Cult, Ch & 1 & 408 \\
\hline Urticaceae & Urtica dioica L. & & Žara, Kopriva, Stinging nettle & $\begin{array}{l}\text { Village \& } \\
\text { shepherd trails }\end{array}$ & Art, $\mathrm{O}$ & 1 & 362 \\
\hline Urticaceae & Urtica dioica L. & & Žara, Kopriva, Stinging nettle & $\begin{array}{l}\text { Village \& } \\
\text { shepherd trails }\end{array}$ & Art, $\mathrm{O}$ & 1 & 362 \\
\hline Urticaceae & Urtica dioica L. & & Žara, Kopriva, Stinging nettle & $\begin{array}{l}\text { Village \& } \\
\text { shepherd trails }\end{array}$ & Art, $\mathrm{O}$ & 5 & 362 \\
\hline Urticaceae & Urtica dioica L. & & Žara, Kopriva, Stinging nettle & $\begin{array}{l}\text { Village \& } \\
\text { shepherd trails }\end{array}$ & Art, $\mathrm{O}$ & 7 & 362 \\
\hline Vitaceae & Vitis vinifera L. & $*$ & Sirće, Vinegar & Cultivated & Cult & 1 & 409 \\
\hline
\end{tabular}

Table 1 details the classification, names, habitat, frequency of use reports and voucher numbers of the 58 species (including 2 subspecies) identified by the Highlanders during this studyAd Adiantetalia, Af Arabidetalia flavescentis, Ag Agrostiealia, Amph Amphoricarpetalia, Arr Arrhenatheretalia, Art Artemisietalia, Atr Atropetalia, Be Brometalia erecti, Bid Bidentetalia, Ch Chenopodietalia, Cor Corynephoretalia, Cult cultivated, Ea Epilobietalia angustifoliae, F Fagetalia, Jun Juniperetalia, M-C MontioCardaminetalia, M Molinietalia, Mol Molinietalia, O Onopordetalia, O-Co Ostryo-Carpinetalia orientalis, Orig Origanetalia, Pm Plantaginetalia majoris, Ps Prunetalia spinosae, Qp Quercetalia pubescentis, S-Ch Scorzonero-Chrysopogonetalia, St Stipetalia, $V$ Vaccinietalia, $V$ - $P$ Vaccinio-Piceetalia

A dagger $(\dagger)$ indicates European endemic taxa. An asterisk $\left(^{*}\right)$ indicates plants found in a garden rather than the wild

and circulatory system disorders. Medium frequency categories included: food, skin/subcutaneous cellular tissue disorders, respiratory system disorders, and illdefined symptoms with many low frequency results (Fig. 4).

The high use of plants for genitourinary system disorders reported (20\%), especially gynaecological uses, may be linked to researcher $L \check{S}$ and the majority of interviewees being female ( $n=14$ of $25,56 \%)$, as women would be more likely to have knowledge or experience of these uses and may also have been more comfortable sharing this information with a woman interviewer. However, high female input was predicted based on our survey of the literature [29].

Panacea treatments were highly cited (14 \%) since they were often cross-listed as food plants, which included a number of economically important plants. High requests for panacea treatments is probably linked to diabetes, which the visiting physician cited as a top health concern. Diabetic insults affect many tissues and organs, leading to symptoms like frequent urination, neuropathy, and slow healing sores, and panacea treatments are an important resource for Highlanders with these symptoms.

Pain treatment was a high frequency usage (13\%), likely due to hard labor in wet and cold conditions. This may also be linked to heart disease which was another top health concern described by the visiting physician. These high frequency categories indicate that Highlander community members are requesting traditional medicines in line with major health concerns.

\section{Frequency of taxa per usage category}

Frequency of taxa per usage category distributions revealed the type of usages of most concern (Fig. 5). The medicinal categories employing the largest number of taxa included genitourinary system disorders, pain, and skin/subcutaneous cellular tissue disorders, indicating that these categories are of high community concern. Medium categories included food, panacea, circulatory system disorders, ill-defined symptoms, respiratory system disorders, and infections/infestations and may mark chronic or recurring health conditions that present more often than low frequency categories (four species or less). Table 2 separates the number of taxa into specific uses in their associated usage category.

High diversity (five or more taxa) was found in the following uses: panacea, internal medicine, heart trouble, genitourinary system health, stomach pain, cuts, and infections.

\section{Informant consensus factor $\left(F_{i c}\right)$ per usage category}

For a disease level analysis, the $F_{i c}$ values were calculated (Fig. 6). Here, 10 of the 15 categories had a $F_{i c}$ value $\geq 0.69$, indicating a high degree of consensus among Lukomir's healers. The average $F_{i c}$ was 0.64 ; to compare human medicinal plant use cross-culturally, non-medicinal categories (bee plants, materials, veterinary aids, food) were removed from this Lukomir survey for an average $F_{i c}$ of 0.76 .

These values are high in comparison to a study in East Timor where the average $F_{i c}$ values calculated for medicinal plant use categories were 0.30 (Laklei 


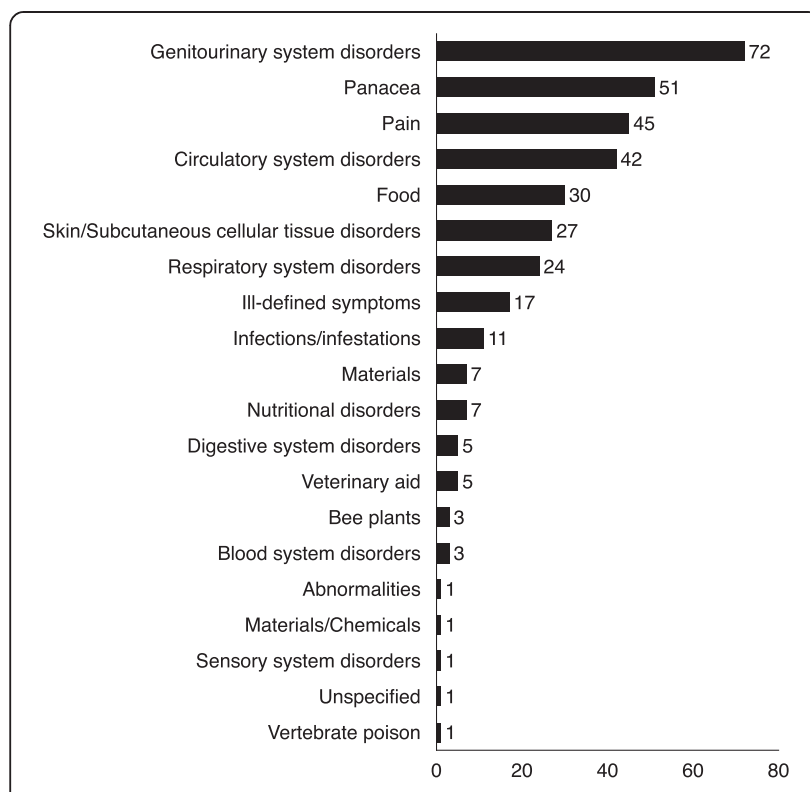

Fig. 4 Distribution of medicinal, food, and material plants according to frequency of use reports

culture) and 0.08 (Idate culture) [30]. The very low value for Idate culture may be due to their culture of secrecy surrounding medicinal plant use. Both Lukomir averages were also greater than averages calculated from a study by Amiguet et al. [31], with the Q'eqchi' Maya of Belize (average $F_{i c}=0.62$ ), and the study with the Yucatec Maya of Mexico $\left(F_{i c}=0.48\right)$ [32]. This difference in $F_{i c}$ results may be explained by recognizing that the Maya informants were from isolated villages while the Lukomir Highlanders, although transhumant, share a single village where they

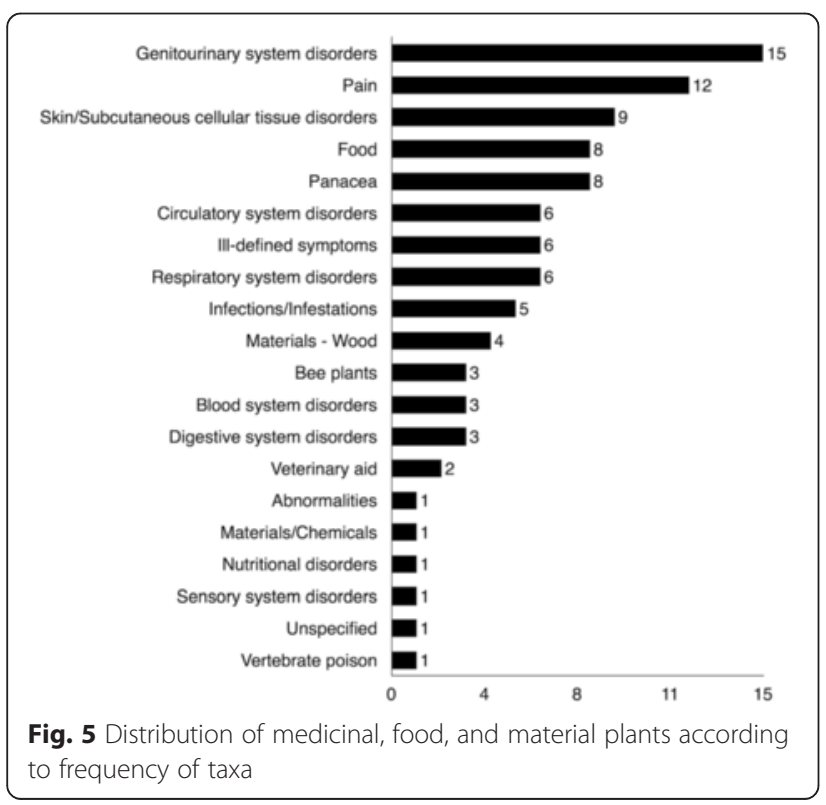

traditionally stayed all winter, snow-locked, and where the interviews were conducted.

These results indicate that these plants are well known in a well defined community-based traditional knowledge system. When consensus is high, these categories are more likely to be active in condition-related pharmacological assays [33].

\section{Analysis of medicinal plant families}

As a first step to identifying key plant families, the number of medicinal, food, and material species by family was ranked (Fig. 7). The three most diverse families were the Lamiaceae (7 species), Asteraceae (6), and Rosaceae (5).

Four medicinal taxa were cross listed as foods, showing that medicinal and food plant categories are not considered mutually exclusive. These traditional foods are often cited for having disease preventing properties and the loss of traditional diets is associated with the rise of chronic conditions such as heart disease and diabetes [1,34].

The phylogeny (Fig. 3) links plant families to their use reports. Among the top three families, Lamiaceae had 80 use reports (77 medicinal) (Table 1). The Lamiaceae in Lukomir flourish in the Mediterranean environment on the south-facing dolomite slopes of the Raktinica Canyon. The Lukomir cultural area is a refuge of wild endemic Lamiaceae taxa, as indicated by our ongoing floristic survey. The Caryophyllaceae family also had high use (36 use reports, all medicinal) (Table 1) and is often found fringing village trails and grasslands. Asteraceae received 32 (30 medicinal) use reports (Table 1), which is not surprising given the family's cosmopolitan distribution throughout Lukomir's alpine, Mediterranean, and continental biogeographical regions.

\section{Traditional medicinal plant preparation}

Leaves are the plant part cited most often by the Highlanders (Fig. 8). Infusions were the most common preparation (Fig. 9), as was found in several other studies of B\&H [6-8]). Some taxa were included in family $\check{c} a j$ (infusion) collections, not necessarily viewed as medicine but consumed after mealtime for their general health benefits [1].

\section{Plant usage compared to other studies in Bosnia \& Herzegovina}

In comparison with other ethnobotanical surveys of medicinal plants used in $\mathrm{B} \& \mathrm{H}$ [6-8]) ten species (including subspecies) in our research have not previously been reported (other than in our previous publication from the same project [1]): Elymus repens (L.) Gould, Euphorbia myrsinites L., Jovibarba hirta (L.) Opiz, Lilium bosniacum (Beck) Fritsch, Matricaria matricarioides (Less.) Porter ex Britton, Phyllitis 
Table 2 The number of taxa for specific uses per usage category

\begin{tabular}{|c|c|}
\hline Usage category & Number of taxa \\
\hline Ailment prophylaxis & 1 \\
\hline \multicolumn{2}{|l|}{ Bee plants } \\
\hline Honey & 3 \\
\hline \multicolumn{2}{|l|}{ Blood system disorders } \\
\hline Blood clots & 1 \\
\hline Circulation & 2 \\
\hline \multicolumn{2}{|l|}{ Circulatory system disorders } \\
\hline Heart trouble & 5 \\
\hline \multicolumn{2}{|l|}{ Digestive system disorders } \\
\hline Diarrhoea & 1 \\
\hline Hard stomach in children & 1 \\
\hline Liver aid & 1 \\
\hline \multicolumn{2}{|l|}{ Food } \\
\hline Vegetables & 4 \\
\hline Beverage & 1 \\
\hline Candy & 1 \\
\hline Preserve, jam & 2 \\
\hline Coffee substitute & 1 \\
\hline \multicolumn{2}{|l|}{ Genitourinary system disorders } \\
\hline Female infertility & 1 \\
\hline Genitourinary system health & 5 \\
\hline Kidney and bladder pain/stones & 2 \\
\hline Urinary tract and kidney health & 4 \\
\hline Heavy or irregular periods & 2 \\
\hline Infection & 3 \\
\hline Urinary tract and kidney infection/cleanser & 1 \\
\hline Vaginal discharge & 2 \\
\hline \multicolumn{2}{|l|}{ III-defined symptoms } \\
\hline Internal medicine & 6 \\
\hline \multicolumn{2}{|l|}{ Infections/infestations } \\
\hline Cold symptoms & 1 \\
\hline Wounds & 1 \\
\hline Fever & 3 \\
\hline \multicolumn{2}{|l|}{ Materials } \\
\hline Flute and violin & 1 \\
\hline Furniture & 1 \\
\hline Rake & 1 \\
\hline Shingle & 1 \\
\hline Tools & 1 \\
\hline \multicolumn{2}{|l|}{ Materials/Chemicals } \\
\hline Teeth cleaning & 1 \\
\hline \multicolumn{2}{|l|}{ Nutritional disorders } \\
\hline Nutritional deficiency/low iron & 1 \\
\hline
\end{tabular}

Table 2 The number of taxa for specific uses per usage category (Continued)

\begin{tabular}{|c|c|}
\hline \multicolumn{2}{|l|}{ Pain } \\
\hline Chest pain & 2 \\
\hline Ear pain (children) & 1 \\
\hline Eye pain & 1 \\
\hline Headache & 2 \\
\hline Menstrual cramps and stomach pain & 1 \\
\hline Pain under ribs & 1 \\
\hline Stomach pain & 5 \\
\hline \multicolumn{2}{|l|}{ Panacea } \\
\hline Cure all & 8 \\
\hline \multicolumn{2}{|l|}{ Respiratory system disorders } \\
\hline Asthma cough and chest pains & 3 \\
\hline Cough/infection & 4 \\
\hline \multicolumn{2}{|l|}{ Skin/Subcutaneous cellular tissue disorders } \\
\hline Condylomata acuminata (warts) & 2 \\
\hline Skin infection and irritations & 1 \\
\hline Cuts and infections & 5 \\
\hline Swollen skin and fat deposits under the skin & 1 \\
\hline \multicolumn{2}{|l|}{ Unspecified } \\
\hline Unspecified & 1 \\
\hline \multicolumn{2}{|l|}{ Vertebrate poison } \\
\hline Chicken poison & 1 \\
\hline \multicolumn{2}{|l|}{ Veterinary aid } \\
\hline Antivenin for sheep & 1 \\
\hline Nit shampoo for sheep & 1 \\
\hline
\end{tabular}

Table 2 presents the number of taxa assigned specific uses and their associated usage category

scolopendrium (L.) Newman, Rubus saxatilis L., Silene uniflora Roth ssp. glareosa (Jord.) Chater \& Walters, Silene uniflora Roth ssp. prostrata (Gaudin) Chater \& Walters, Smyrnium perfoliatum L. (Table 3). New medicinal uses not reported in any of the aforementioned systematic surveys were cited for a total of 29 species, including the ten newly reported species.

\section{Use of rare and endemic plants}

This study considers endemics in the context of Flora Europaea and did not consider garden plants as endemics. Garden plants listed in our survey, such as potato, have been denoted in Table 1. It was observed that Lukomir is a wild refuge for many european endemics. For example, sage was never found in the garden, and was only located once, halfway down Europe's largest canyon.

The Highlanders use eight endemic plant species: Helleborus odorus Waldst. et Kit., Gentiana lutea L., Lilium bosniacum (Beck) Fritsch, Silene uniflora Roth 


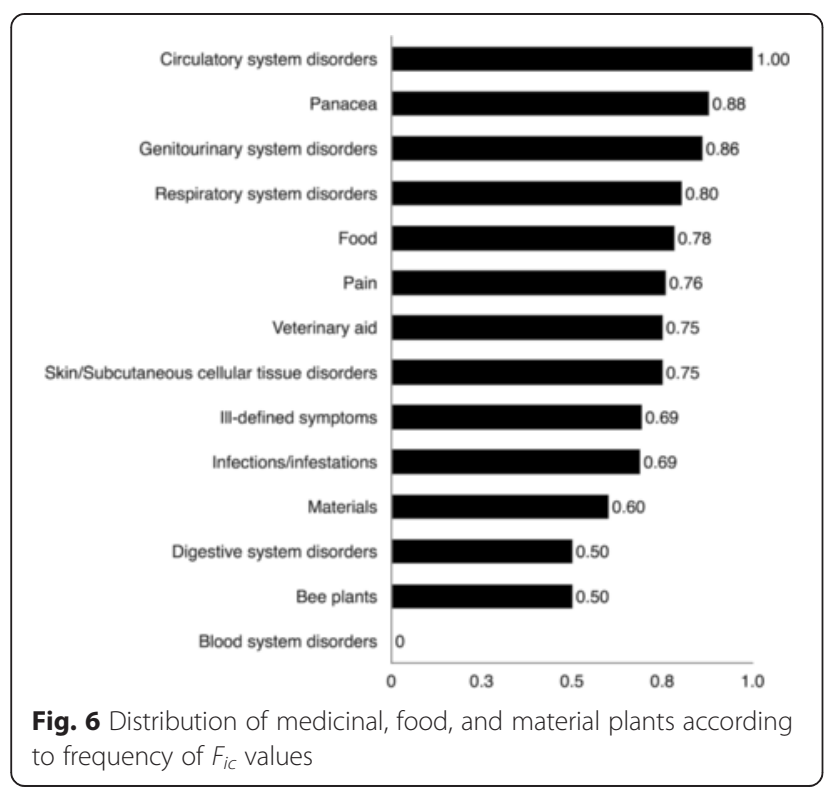

ssp. glareosa (Jord.) Chater \& Walters., Silene uniflora Roth ssp. prostrata (Gaudin) Chater \& Walters, Salvia officinalis L., Jovibarba hirta (L.) Opiz, and Satureja montana L. Of the total medicinal plant use, $13 \%$ is endemic flora. This is not surprising, since the Lukomir Highlanders are an indigenous community inhabiting one of Europe's most diverse biological areas $[5,6]$.

Lilium bosniacum, Gentiana lutea L., Silene uniflora Roth ssp. prostrata (Gaudin) Chater \& Walters and Jovibarba hirta (L.) Opiz, are among the species which have not been listed in previous traditional medicine research in B\&H (Table 1).

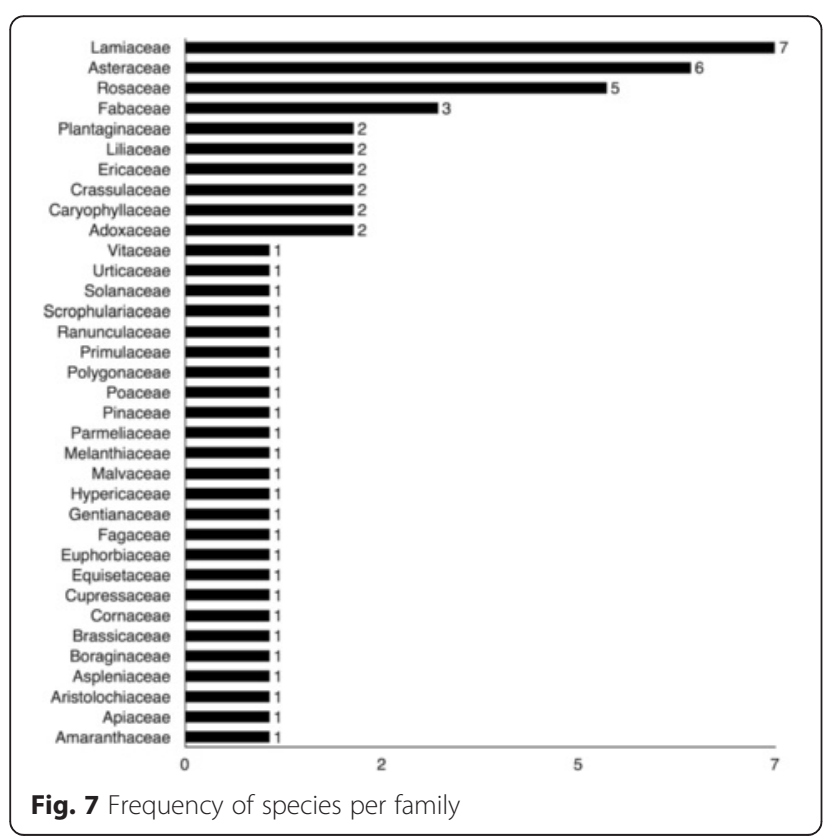

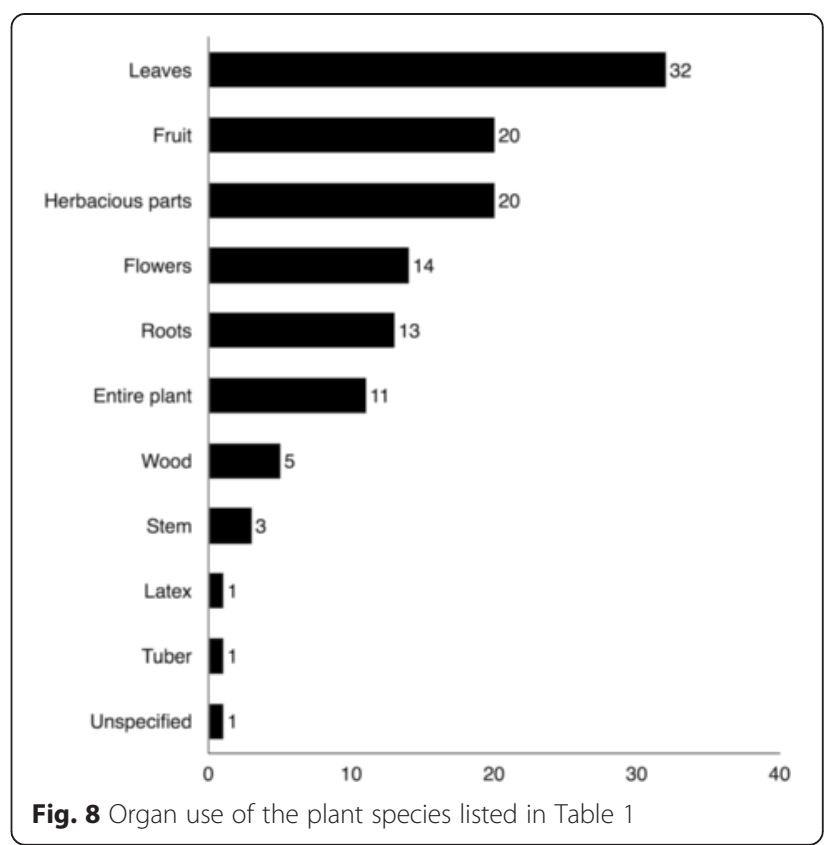

The Highlanders uses the root of Gentiana lutea L. have not been reported in other studies in the country (Table 1).

Lilium bosniacum (Beck) Fritsch, the Bosnian Lily, is endemic to the central Dinaric Alps whose taxonomic status has been much debated [35]. Studies have come to differing conclusions as to whether L. bosniacum is a distinct species, a subspecies or a variety of $L$. carniolicum [35-37]). Recent molecular cytogenic studies have found evidence to support $L$. bosniacum's status as a distinct species [35, 37]. According to Tropicos, Lilium bosniacum (Beck) Fritsch has species rank [38].

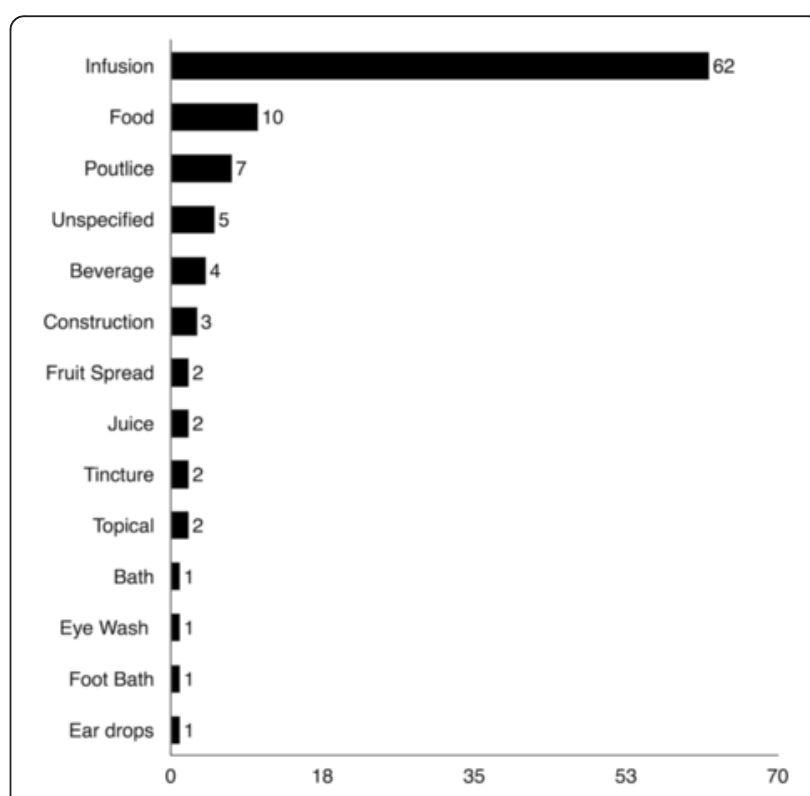

Fig. 9 Plant preparation reports of the plant species listed in Table 1 
Table 3 A comparison of this study's reported plants with other ethnobotanical studies in Bosnia and Herzegovina

\begin{tabular}{|c|c|}
\hline Genus species & $\begin{array}{l}\text { Plant usage reported in } \\
\text { other studies }\end{array}$ \\
\hline Achillea millefolium $\mathrm{L}$. & $Y[8,29,39]$ \\
\hline Anthyllis vulneraria $\mathrm{L}$. & $Y[8,29,39]$ \\
\hline Artemisia absinthium L. & $Y[6-8,29]$ \\
\hline Asarum europaeum L. & $\mathrm{Y}[8]$ \\
\hline Capsella bursa-pastoris (L.) Medik. & $Y[6,8,29,39]$ \\
\hline Cetraria islandica (L.) Ach & Y [6-8] \\
\hline Chenopodium bonus-henricus $\mathrm{L}$. & Y [39] \\
\hline Cichorium intybus L. & $Y[6,8,29,39]$ \\
\hline Convallaria majalis L. & $\mathrm{Y}[8]$ \\
\hline Cornus mas L. & $Y[6,8,39]$ \\
\hline Crataegus monogyna Jacq. & $Y[6-8,29,39]$ \\
\hline Elymus repens (L.) Gould & N \\
\hline Equisetum arvense $\mathrm{L}$. & $\mathrm{Y}[8,29]$ \\
\hline Euphorbia myrsinites L. & N \\
\hline Fagus sylvatica $\mathrm{L}$. & $\mathrm{Y}[8,39]$ \\
\hline Gentiana lutea L. & $\mathrm{Y}[6,8]$ \\
\hline Helleborus odorus Waldst. \& Kit. ex Willd. & $\mathrm{Y}[8]$ \\
\hline Hypericum perforatum $\mathrm{L}$. & $Y[6-8,29,39]$ \\
\hline Jovibarba hirta (L.) Opiz & $\mathrm{N}$ \\
\hline Juniperus communis $\mathrm{L}$. & Y [6-8] \\
\hline Lilium bosniacum (Beck) Fritsch & $\mathrm{N}$ \\
\hline $\begin{array}{l}\text { Matricaria matricarioides (Less.) Porter ex } \\
\text { Britton }\end{array}$ & N \\
\hline Mentha longifolia (L.) L. & $Y[6-8,39]$ \\
\hline Mentha pulegium $\mathrm{L}$. & $Y[6-8,29,39]$ \\
\hline Nepeta cataria L. & $Y[6,8,29]$ \\
\hline Ononis spinosa $\mathrm{L}$. & $Y[6-8,39]$ \\
\hline Phyllitis scolopendrium (L.) Newman & $\mathrm{N}$ \\
\hline Plantago lanceolata $\mathrm{L}$. & $Y[6-8,39]$ \\
\hline Plantago major L. & $Y[6-8,39]$ \\
\hline Polygonum bistorta $\mathrm{L}$. & $Y[7,8,39]$ \\
\hline Primula veris $\mathrm{L}$. & $\mathrm{Y}[8,39]$ \\
\hline Prunus spinosa $\mathrm{L}$. & $Y[7,8,39]$ \\
\hline Rosa glauca Pourr. & Y [39] \\
\hline Rubus idaeus L. & $Y[6-8,39]$ \\
\hline Rubus saxatilis $\mathrm{L}$. & $\mathrm{N}$ \\
\hline Salvia officinalis $\mathrm{L}$. & $Y[6-8,29,39]$ \\
\hline Sambucus ebulus L. & $Y[6,7,29]$ \\
\hline Sambucus nigra L. & $Y[6-8,39]$ \\
\hline Satureja montana L. & $Y[6-8,29,39]$ \\
\hline Sedum sexangulare $\mathrm{L}$. & Y [29] \\
\hline Silene uniflora Roth ssp. glareosa (Jord.) & N \\
\hline
\end{tabular}
Chater \& Walters
Table 3 A comparison of this study's reported plants with other ethnobotanical studies in Bosnia and Herzegovina (Continued)

\begin{tabular}{ll}
\hline Silene uniflora Roth ssp. prostrata (Gaudin) & $\mathrm{N}$ \\
Chater \& Walters & $\mathrm{N}$ \\
Smyrnium perfoliatum L. & $\mathrm{Y}[6,7]$ \\
Solanum tuberosum L. & $\mathrm{Y}[6-8,29,39]$ \\
Symphytum officinale L. & $\mathrm{Y}[6-8,29,39]$ \\
Taraxacum officinale F.H. Wigg. & $\mathrm{Y}[6-8,29]$ \\
Teucrium montanum L. & $\mathrm{Y}[8,29,39]$ \\
Thymus serpyllum L. & $\mathrm{Y}[6-8]$ \\
Tilia platyphyllos Scop. & $\mathrm{Y}[6,7,39]$ \\
Trifolium pratense L. & $\mathrm{Y}[6,7,39]$ \\
Trifolium repens L. & $\mathrm{Y}[6-8,29,39]$ \\
Tussilago farfara L. & $\mathrm{Y}[6-8,29,39]$ \\
Urtica dioica L. & $\mathrm{Y}[6-8,39]$ \\
Vaccinium myrtillus L. & $\mathrm{Y}[6,8,39]$ \\
Vaccinium vitis-idaea L. & $\mathrm{Y}[8]$ \\
Veratrum album L. & $\mathrm{Y}[7,8]$ \\
Verbascum thapsus L. & $\mathrm{Y}[6,7]$ \\
Vitis vinifera L. &
\end{tabular}

Table 3 presents which plants have been cited in other studies in B\&H

Lilium bosniacum has been classified as a rare and vulnerable taxon in the pending edition of the Red Book of B\&H. Despite not being its typical habitat, L. bosniacum is found in Bjelašnica [35]. One informant in Lukomir cited two uses for L. bosniacum.

Salvia officinalis L. (Kadulja, sage) was not found in gardens, but growing wild. In nearby Albania, this plant is an important export product to Western Europe and the United States [39]. This plant was cited a large number of times in the 2007 systemic study of the country's plant use [8].

\section{Post Bosnian War development, conservation, and the dietary shift in Lukomir}

Recent changes have deteriorated the health and environment status in Lukomir. Before 1997, three vodenica mlini (hydro powered cereal mills) conducted the process of removing hard seed coats from Lukomir's cereal crops. The Vodenica waterway runs from the peak of Obalj mountain and down through the canyons of the Rakitnica (Fig. 2). Troughs handcrafted from the hardwood Fagus sylvatica L. (Bukva, Beech) funneled the vodenica and hydro powered the wooden turbines constructed of F. sylvatica vanes and axils (Fig. 10). The turbine turned one of the two hand fashioned grinding stones that produced wholegrain cereal flour. Our informants indicated that the cereals routinely grown and milled in Lukomir were: Avena sativa L. (Zob, Oat), Secale sereale L. (Raž, Rye), Hordeum vulgare L. (Ječam, 
Barley), Triticum aestivum L. (Pšenica, Wheat), and Zea mays L. (Kukuruz, Corn).

Once powered by the spring rains and snowmelt, the mills are now in disuse on the edge of Donji Lukomir (see M, Fig. 2). The local cereal crops are no longer cultivated and milled and have been replaced by imported soft white wheat flour. When asked why the mlini are not used, informants explained that in 1997, 1 year after the Bosnian War, water diversion began in Lukomir. Water pipelines were placed at the mouth of the Obalj mountain spring to share water with lower elevation communities in the Municipality of Konjic. Following diversion, the waterways began to dry up by mid-June, leaving the mills devoid of running water. There was no hydro power available for milling the June, July, and September cereal harvests. This water work development caused the collapse of the vodenica mlini, cultivation of indigenous cereal landraces, and Lukomir's organic multigrain diet. As running the mills involved considerable physical effort, a source of exercise was also lost.

Water work diversion lead to a community-wide dietary shift. Many locals now depend on a food truck that delivers white flour once per week. The time spent working to produce their own multigrain flour is gone, and the diet and lifestyle shift is contributing to
Lukomir's greatest health concerns: heart disease and diabetes [1].

All of the rare and endemic flora and many other species listed in (Table 1) are found in the area from Obajl (1874 $\mathrm{m}$ a.s.l.) to the Rakitnica River (640 m a.s.l.). These areas are now more endangered because of the water work diversion and ongoing habitat loss. Although all people from $B \& H$ should have equal and shared access to water, this particular development was placed in the centre of one of the most biogeographically sensitive areas of both the country and continent without sufficient consultation and foresight.

The collapse of Lukomir's traditional food system comes at a time when tourism is increasing in the community and B\&H is modelling itself as an international destination to provide an experience of European traditional culture. The region's flora and organic multigrain products are popular attractions among hiking enthusiasts and health conscious visitors to the Dinaric Alps. Many women in the community depend on tourism as they open their homes to tourists, serve food, teas, and coffee, and sell their knitted merchandise. Planning for a self-sufficient future for the Lukomir Highlanders should include preservation of the vodenica mlini as a unique cultural technology, an attraction for visitors to the area and a contributor to a healthier lifestyle.
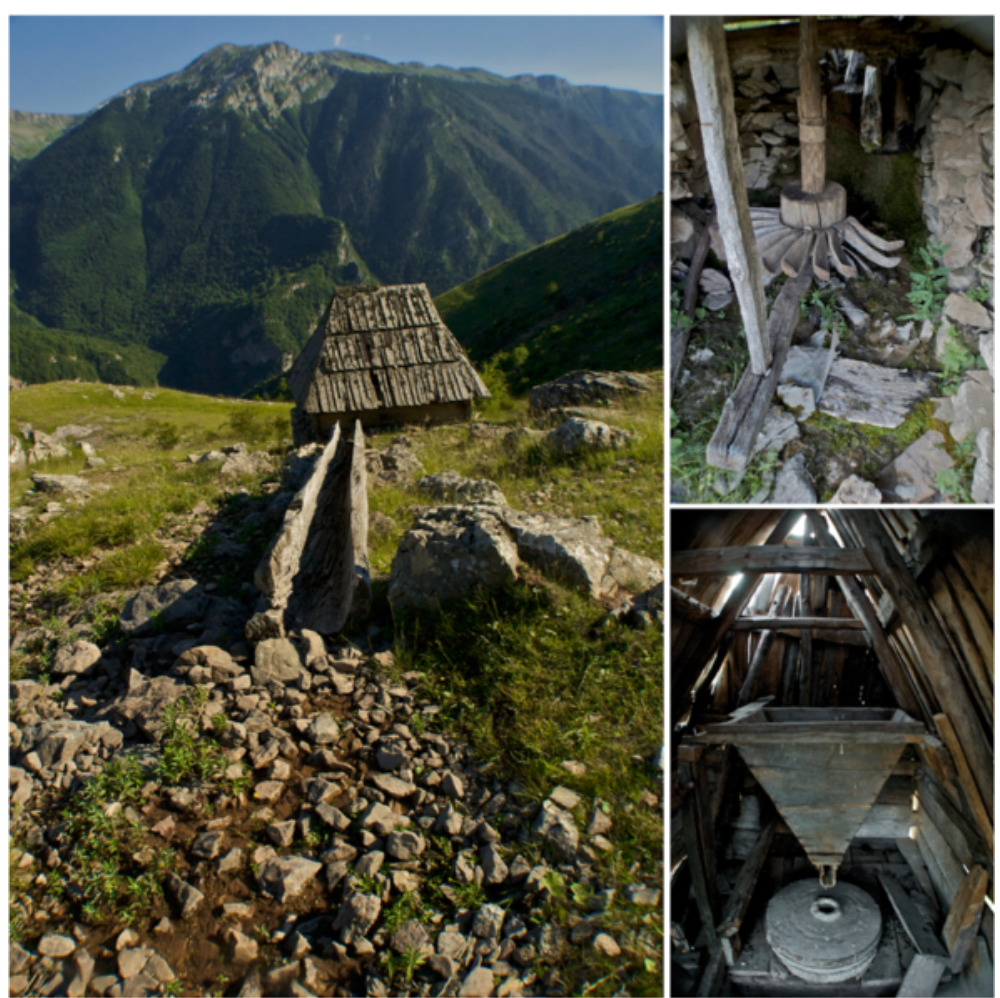

Fig. 10 A vodenica mlini (hydro mill) on the dried up Vodenica waterway in July 


\section{Conclusions}

Although post war development has contributed to the erosion of the self-sustaining traditional lifestyle of the Lukomir Highlanders, our results demonstrate that they continue to have a strong traditional medicine and gathered food system. This traditional knowledge must continue to be valued and maintained in planning for a durable, self-sufficient future for the Lukomir Highlanders. In addition, special emphasis should be placed on the preservation of the vodenica mlini (hydro cereal mills) - a unique cultural technology and visitor attraction that contributes to a traditionally healthy diet and lifestyle.

\section{Abbreviations \\ B\&H: Bosnia and Herzegovina; DL: Donji Lukomir; GL: Gornji Lukomir; CIDA: Canadian International Development Agency; BH MAC: Bosnia and Herzegovina Mine Action Centre; $F_{i c}$ Informant consensus factor.}

\section{Competing interests}

The authors declare that they have no competing interests.

\section{Authors' contributions}

JF: conceived project, funded project, established collaboration, designed and carried out ethnobotany, plant ID, and produced text, figures and tables. LŠ: established collaboration, conceived project, funded project, designed and carried out ethnobotany, assisted in writing and producing tables. ECHC: assisted in writing and producing figures. RLG: assisted in research, writing and producing tables and figures. ST: participated in design, local collaboration, plant ID, logistics and helped to draft the manuscript. AC: funded project, participated in design, coordination and helped to draft the manuscript. MJB: participated in design, coordination and helped to draft the manuscript. EA: funded project, participated in design, coordination and helped to draft the manuscript. SR: funded project, established local collaboration, participated in design, coordination, plant ID, and helped to draft the manuscript. JTA: funded project, participated in design, coordination and helped to draft the manuscript. All authors read and approved the final manuscript.

\section{Acknowledgements}

We would like to thank the Lukomir Highlanders for their hospitality, guidance, and for sharing their knowledge. We thank the Municipality of Konjic, Foundation GEAt, and the University of Sarajevo, Department of Biology for logistic support. Many thanks to JF's doctoral committee members Nancy Turner, Naomi Cappucino, Christiane Charest, Alexandre Poulain, and Julian Starr. We thank Ammar Saleem for laboratory assistance. We thank members of GEA+, Kujundzić Family, Alikadić family, Elivra Jahić, Sedik Velić and Adnan Šačiragić for photography, field work, and translation assistance. We thank Emcarta Inc. for funding editorial services by Sonja Campbell and the research assistant position of RLG.

JF and LŠ were supported by the Canadian International Development Agency (Association of Universities and Colleges Canada, Partnerships for Tomorrow Program, Phase II) travel grant. JF was supported by National Science and Engineering Research Council of Canada's (NSERC) Alexander Graham Bell Post Graduate Scholarship, Mitacs Elevate Postdoctoral Fellowship, and Indspire. JTA acknowledges NSERC discovery funding.

\section{Author details}

${ }^{1}$ Department of Biology, University of Ottawa, Gendron Hall Room 160, 30 Marie Curie, Ottawa K1N 6 N5ON, Canada. ${ }^{2}$ Institute of Economic Botany, The New York Botanical Garden, 200th Street and Kazimiroff Blvd, The Bronx, NY 10458-5126, USA. ${ }^{3}$ Department of Chronic Diseases, OHRI The Ottawa Hospital, University of Ottawa OBGYN Suite 8425, 501 Smyth Rd, Ottawa K1Y 4E9ON, Canada. ${ }^{4}$ Emcarta Inc., 32 Lewis Street, Ottawa K2P 0S3ON, Canada. ${ }^{5}$ Department of Obstetrics and Gynecology, University of Ottawa / the Ottawa Hospital, 501 Smyth Road, Ottawa K1H 8 L6ON, Canada. ${ }^{6}$ Department of Botany, Center of Ecology and Natural Resources, University of Sarajevo,
Hrasnička cesta 15, 71210 Sarajevo, Bosnia and Herzegovina. ${ }^{7}$ Université de Montréal, Institut de recherche en biologie végétale, Jardin botanique de Montréal 4101 rue Sherbrooke Est, Montréal H1X 2B2QC, Canada. ${ }^{8}$ Foundation GEA+, Kranjčevićeva 41, 71000 Sarajevo, Bosnia and Herzegovina.

Received: 5 November 2015 Accepted: 6 November 2015 Published online: 25 November 2015

\section{References}

1. Ferrier J, Šačiragić L, Chen ECH, Trakić S, Saleem A, Alikadić E, et al. Ways the Lukomir Highlanders of Bosnia and Herzegovina treat diabetes. In: Pieroni A, Quave $\mathrm{CL}$, editors. Ethnobotany and biocultural diversities in the Balkans. New York: Springer; 2014. p. 13-28. http://www.bit.ly/1LaXY4B Accessed 18 Oct 2015.

2. Nikolić S, Šarančić Logo A. Lukomir. Tradition and change in a Bosnian Village. Vernac Archit. 2011;42:53-66.

3. Marjanović D, Kapur L, Drobnic K, Budowle B, Pojskic N, Hadziselimovic R. Comparative study of genetic variation at 15 STR loci in three isolated populations of the Bosnian mountain area. Hum Biol Int Rec Res. 2004;76:15-31

4. Tourism Association of Federation of Bosnia and Herzegovina. Lukomir Umoljani Sarajevo Region. 2005. http://www.bhtourism.ba/eng/lukomir. wbsp Accessed 27 July 2014.

5. European Environment Agency. Biodiversity 'hot spots' in the pan-European region. http://www.eea.europa.eu/data-and-maps/figures/biodiversity-hotspotsin-the-pan-european-region Accessed 23 May 2013.

6. Šarić-Kundalić B, Dobeš C, Klatte-Asselmeyer V, Saukel J. Ethnobotanical survey of traditionally used plants in human therapy of east, north and north-east Bosnia and Herzegovina. J Ethnopharmacol. 2011;133:1051-76.

7. Šarić-Kundalić B, Dobeš C, Klatte-Asselmeyer V, Saukel J. Ethnobotanical study on medicinal use of wild and cultivated plants in middle, south and west Bosnia and Herzegovina. J Ethnopharmacol. 2010;131:33-55.

8. Redžić S. The ecological aspect of ethnobotany and ethnopharmacology of population in Bosnia and Herzegovina. Coll Antropol. 2007;31:869-90.

9. Pieroni A, Quave $C L$, editors. Ethnobotany and biocultural diversities in the Balkans. New York: Springer; 2014.

10. Pieroni A, Dibra B, Grishaj G, Grishaj I, Maçai SG. Traditional phytotherapy of the Albanians of Lepushe, Northern Albanian Alps. Fitoterapia. 2005;76:379-99.

11. Pieroni A. Local plant resources in the ethnobotany of Theth, a village in the Northern Albanian Alps. Genet Resour Crop Evol. 2008;55:1197-214.

12. Pieroni A, Nedelcheva A, Hajdari A, Mustafa B, Scaltriti B, Cianfaglione K, et al. Local knowledge on plants and domestic remedies in the mountain villages of Peshkopia (Eastern Albania). J Mt Sci. 2014;11:180-93.

13. Menković N, Šavikin K, Tasić S, Zdunić G, Stešević D, Milosavljević S, et al Ethnobotanical study on traditional uses of wild medicinal plants in Prokletije Mountains (Montenegro). J Ethnopharmacol. 2011;133:97-107.

14. Mustafa B, Hajdari A, Pajazita Q, Syla B, Quave CL, Pieroni A. An ethnobotanical survey of the Gollak region, Kosovo. Genet Resour Crop Evol. 2012:59:739-54.

15. Jarić S, Popović Z, Mačukanović-Jocić M, Djurdjević L, Mijatović M, Karadžić $B$, et al. An ethnobotanical study on the usage of wild medicinal herbs from Kopaonik Mountain (Central Serbia). J Ethnopharmacol. 2007;111:160-75.

16. Zlatković BK, Bogosavljević SS, Radivojević AR, Pavlović MA. Traditional use of the native medicinal plant resource of Mt. Rtanj (Eastern Serbia): ethnobotanical evaluation and comparison. J Ethnopharmacol. 2014;151:704-13.

17. Ivancheva S, Stantcheva B. Ethnobotanical inventory of medicinal plants in Bulgaria. J Ethnopharmacol. 2000;69:165-72.

18. Kultur S, Sami S. Medicinal plants used in Isperih (Razgrad-Bulgaria) district. Turkish J Pharm Sci. 2009;6:107-24.

19. Rexhepi B, Mustafa B, Hajdari A, Rushidi-Rexhepi J, Quave CL, Pieroni A. Traditional medicinal plant knowledge among Albanians, Macedonians and Gorani in the Sharr Mountains (Republic of Macedonia). Genet Resour Crop Evol. 2013;60:2055-80.

20. Pieroni A, Rexhepi B, Nedelcheva A, Hajdari A, Mustafa B, Kolosova V, et al. One century later: the folk botanical knowledge of the last remaining Albanians of the upper Reka Valley, Mount Korab, Western Macedonia. J Ethnobiol Ethnomed. 2013;9:22. 
21. European Environment Agency. Natura 2000. Biogeographic regions of Europe. 2012

22. Thomas E, Vandebroek I, Van Damme P. What works in the field? A comparison of different interviewing methods in ethnobotany with special reference to the use of photographs. Econ Bot. 2007;61:376-84.

23. The Bosnia and Herzegovina Mine Action Centre. Bjelašnica area map. [http://trekkingbosnia.com/en/images/map/bjelasnica_mines.jpg] Accessed 18 Oct 2015.

24. Trotter R, Logan M. Informant consensus: a new approach for identifying potentially effective medicinal plants. In: Etkin N, editor. Plants in indigenous medicine and diet: biobehavioural approaches. Bedford Hills: Redgrave Publishers; 1986. p. 91-112.

25. Domac R. Mala Flora Hrvatske I Susjednih Područja. Školska Knijga: Zagreb; 1984.

26. Missouri Botanical Garden. Tropicos. http://www.tropicos.org/ Accessed 10 Oct 2013.

27. Angiosperm Phylogeny Group. An update of the Angiosperm Phylogeny Group classification for the orders and families of flowering plants: APG III. Bot J Linn Soc. 2009;161:105-21.

28. Ferrier J. Ethnobotany, Pharmacology, and Metabolomics of Antidiabetic Plants used by the Eeyou Istchee Cree, Lukomir Highlanders, and Q'eqchi' Ma-ya. University of Ottawa: The New York Botanical Garden; 2014. http://bit.ly/10ETqbH Accessed 18 Oct 2015

29. Redžić S. Wild medicinal plants and their usage in traditional human therapy (Southern Bosnia and Herzegovina, W. Balkan). J Med Plants Res. 2010;4:1003-27.

30. Collins S, Martins X, Mitchell A, Teshome A, Arnason JT. Quantitative ethnobotany of two east timorese cultures. Econ Bot. 2006:60:347-61.

31. Amiguet V, Arnason JT, Maquin P, Cal V, Vindas P, Poveda L. A consensus ethnobotany of the Q'eqchi' Maya of Southern Belize. Econ Bot. 2005:59:29-42.

32. Heinrich M. Ethnobotany and its role in drug development. Phytother Res. 2000;14:479-88.

33. Leaman D, Arnason JT, Yusuf R, Sangat-Roemantyo $H$, Soedjito $H$ Angerhoferc C. Malaria remedies of the Ken- yah of the Apo Kayan, West Kalimantan, Indonesian Borneo: a quantitative assessment of local consensus as an indicator of biological efficacy. J Ethnopharmacol. 1995:49:1-16

34. Johns T, Chapman L. Phytochemicals ingested in traditional medicines as modulators of energy metabolism. In: Arnason JT, Mata R, Romeo J, editors. Phytochemistry of medicinal plants. New York: Springer; 1995. p. 161-88.

35. Muratović E, Bogunić F, Šoljan D, Siljak-Yakovlev S. Does Lilium bosniacum merit species rank? A classical and molecular-cytogenetic analysis. Plant Syst Evol. 2005;252:97-109.

36. Rešetnik I, Liber Z, Satovic Z, Cigić P, Nikolić T. Molecular phylogeny and systematics of the Lilium carniolicum group (Liliaceae) based on nuclear ITS sequences. Plant Syst Evol. 2007;265:45-58.

37. Muratović E, Robin O, Bogunić F, Šoljan D, Siljak-Yakovlev S. Karyotype evolution and speciation of European lilies from Lilium sect. Liriotypus Taxon. 2010;59:165-75.

38. Missouri Botanical Garden. Tropicos: Lilium bosniacum (Beck) Fritsch http://tropicos.org/Name/100215787 Accessed 18 October 2015

39. Pieroni A. People and plants in lëpushë traditional medicine, local foods and post-communism in a Northern Albanian Village. In: Pardo-deSantayana M, Pieroni A, Puri R, editors. Ethnobotany in the new europe: people, health and wild plant resources. Bergham Books; New York 2010. p. 17-48.

\section{Submit your next manuscript to BioMed Central and take full advantage of:}

- Convenient online submission

- Thorough peer review

- No space constraints or color figure charges

- Immediate publication on acceptance

- Inclusion in PubMed, CAS, Scopus and Google Scholar

- Research which is freely available for redistribution 